\title{
Klein-Gordon equations for horizontal transverse oscillations in two-dimensional coronal loops
}

\author{
Qiang $\mathrm{Hu}^{1}$, J. F. McKenzie ${ }^{1,2,3}$, and G. M. Webb ${ }^{1}$ \\ 1 CSPAR, University of Alabama, Huntsville, AL, USA \\ e-mail: qh0001@uah.edu \\ 2 Department of Mathematics and Statistics, Durban University of Technology, Steve Biko Campus, Durban, South Africa \\ ${ }^{3}$ School of Mathematical Sciences, University of KwaZulu-Natal, Durban, South Africa
}

Received 6 June 2011 / Accepted 14 March 2012

\begin{abstract}
We present a theory for hydromagnetic waves in an axi-symmetric background magnetic field in which the wave equations for the horizontal transverse magnetic field and velocity perturbations can be transformed into Klein-Gordon (KG) equations. For harmonic time variations, the KG equations become a set of ordinary differential equations that can be solved along any individual field line, subject to boundary conditions at the two ends. The solutions provide the spatial (latitudinal) profiles of the transverse magnetic field and velocity oscillations, especially in the horizontal direction, along the field line. In particular, we examine the KG solutions for two background field geometries: a local dipole field line, and a stretched global dipole field line which may approximate coronal loop geometries in the solar corona. The results yield the oscillation frequencies in agreement with observations (periods on the order of minutes), and the spatial profiles which are characteristic of a propagating type near the center of the loop and a possible evanescent type towards the footpoints of the loop. The latter solution arises when the oscillation frequency is less than a critical cut-off frequency which varies spatially along the loop. The oscillation amplitude is also affected by an adiabatic growth/decay factor along the loop. We discuss the implications of our results and future applications to coronal loop oscillations.
\end{abstract}

Key words. Sun: corona - Sun: magnetic topology - Sun: oscillations

\section{Introduction}

Coronal loop oscillations have been observed for decades in space by direct remote-sensing imaging of EUV emissions and the Doppler shift signatures in certain spectral lines (Aschwanden et al. 2002; Schrijver et al. 2002; Wang et al. 2002, 2003), among other means (Aschwanden 2004; Nakariakov \& Verwichte 2005). Especially with the advent of the Transition Region and Coronal Explorer (TRACE) satellite, systematic and comprehensive observational studies as first carried out by Aschwanden et al. (2002) and Schrijver et al. (2002) are made available and are more reliable. Those authors reported a set of observations of transverse coronal loop oscillations and an analysis of their geometric and oscillatory characteristics. In particular, they reported the oscillation periods in the range of $P=2-33 \mathrm{~min}$ (average about $5.4 \mathrm{~min}$ ), and a lower limit of the loop density in the range of $0.13-1.7 \times 10^{9} \mathrm{~cm}^{-3}$, together with other loop oscillation parameters, such as the transverse amplitude, the loop half length based on a circular loop model, and decay times. These observations, especially the oscillation periods, have generally been related to fast kink-mode magnetohydrodynamic (MHD) waves, based on linearized MHD equations for a straight slab or cylindrical geometry of a coronal loop (Aschwanden 2004; Nakariakov \& Verwichte 2005, and references therein). The kink modes are believed to possess "vertical", as well as "horizontal" mode (e.g., Wang et al. 2008). The "vertical" mode indicates oscillations in the loop plane, and the "horizontal" mode indicates oscillations perpendicular to the loop plane.
A number of theoretical works have been proposed to examine various aspects of loop oscillation characteristics, and the underlying mechanisms for wave excitation and damping (e.g., Terradas et al. 2006; Van Doorsselaere et al. 2004; Nakariakov \& Verwichte 2005). A special issue of Space Sci. Rev. (vol. 149, 2009) contains a collection of review articles on the subject of solar coronal seismology related to loop oscillations. In particular, Ruderman \& Erdélyi (2009) provided a comprehensive review of the theory of transverse oscillations in coronal loops concerning fast kink waves in a magnetic flux tube of finite radius and various effects. Van Doorsselaere et al. (2009) reviewed both the theory and numerical simulations on the effect of loop curvature on kink oscillations, including curved slab and curved cylinder models of the coronal loops. Other works have also included the curvature of a loop in either 1D or 2D geometry, and even non-planar loops (Ruderman \& Scott 2011). Rigorous 3D MHD simulations have also been performed (e.g., Miyagoshi et al. 2004; Ofman 2009; Selwa \& Ofman 2010).

To complement the foregoing works, here we report on a simple theory of transverse hydromagnetic waves that was developed and applied earlier to Earth's dipole magnetic field (McKenzie \& Hu 2010; Webb et al. 2012). We present the theory as an alternative model of transverse (toroidal) coronal loop oscillation (applicable to the aforementioned "horizontal" mode) in an axi-symmetric background magnetic field. In the spherical coordinates, $(r, \theta, \phi)$, a background magnetic field line (loop) geometry is prescribed in the $(r, \theta)$ plane, such as a dipole to be discussed later, and the oscillation is along the $\phi$ direction (and $\partial / \partial \phi=0)$. In the present study, we concentrate on the theoretical investigation of qualitative mode identification, and quantitative 
comparison of oscillation frequency/periods with observations only, under general solar coronal conditions.

The paper is organized as follows: in the next section, we discuss the background equilibrium state and the governing equations of the perturbations. Then we show the wave equations of the toroidal perturbations of both the magnetic field and velocity, that can be transformed into a special form of ordinary differential equations. We then apply the equations to the coronal loop oscillations subject to two background magnetic field geometries that may mimic a real closed loop geometry in the solar corona in Sect. 4. We show solutions of the equations in terms of oscillation frequencies and wave forms for different modes of ascending orders, subject to a set of boundary conditions. Lastly we summarize our results and discuss future applications of our model.

\section{Background equilibrium state}

The equilibrium state of the background plasma and magnetic field under investigation is governed by the ideal MHD equations, without mean background flow. Therefore the set of equations is given by the momentum equation and the Maxwell's equations satisfied by the magnetic field. Under the assumption of axi-symmetry in the spherical coordinate $(\partial / \partial \phi=0)$, we consider toroidal wave perturbations $\left(b_{\phi}, u_{\phi}\right)$ in the magnetic field and fluid velocity in a background magnetic field $\boldsymbol{B}_{0}=\left(B_{r}, B_{\theta}, 0\right)$. The corresponding electric field becomes

$\boldsymbol{E}=-\boldsymbol{u} \times \boldsymbol{B}=-u_{\phi} B_{r} \hat{\theta}+u_{\phi} B_{\theta} \hat{r}$.

The only non-vanishing $\phi$ component of Faraday's law and the $\phi$ component of the momentum equation yield, respectively,

$\frac{\partial b_{\phi}}{\partial t}=\frac{B_{\theta}}{r} \frac{\mathrm{d} u_{\phi}}{\mathrm{d} \theta}+\frac{u_{\phi} B_{\theta}}{r} \frac{1}{l_{b}}$,
$\frac{\partial u_{\phi}}{\partial t}=\frac{B_{\theta}}{\rho r} \frac{\mathrm{d} b_{\phi}}{\mathrm{d} \theta}+\frac{b_{\phi} B_{\theta}}{\rho r} \frac{1}{l_{u}}$,

where all spatially dependent variables are functions of the single coordinate $\theta$ only, along an individual field line (Webb et al. 2012). Here the function $\frac{1}{l_{u}}=-\frac{1}{l_{b}}=\frac{\mathrm{d}}{\mathrm{d} \theta} \ln (r \sin \theta)$ is evaluated along the field line and is determined by the background magnetic field topology.

In these equations, there is no contribution from the pressure gradient term, nor the gravity, because of the assumed axisymmetry, i.e., $\partial / \partial \phi=0$. However in the other dimensions, i.e., in the $(r, \theta)$ plane, under the linear wave approximation, the force balance is such that both the pressure gradient and gravity forces counter the Lorentz force. In perhaps the simplest but yet realistic background magnetic field geometry of a dipole (a potential field) as illustrated in Fig. 1a where several field lines of different apex heights from the solar surface and different locations of their footpoints are drawn, the usual hydrodynamic equilibrium $\nabla p=\rho \boldsymbol{g}$ is maintained. This case is to be examined in Sect. 4.1. Whereas in Sect. 4.2, we examine a more general stretched dipole field configuration, as illustrated in Fig. 1b of one particular loop, that maintains a magnetohydrostatic equilibrium with non-vanishing Lorentz force (Gibson \& Low 1998).

We note that our approach is focused on modeling the magnetic field line oscillations. We are aware that from observations, the bright loop structures are mostly representative of enhanced plasma density features (e.g., Aschwanden 2004). Therefore the coronal loops are often assumed to be of finite cross-section areas with magnetic field embedded. In the current investigation, we do not explicitly incorporate such a finite-column density structure. Nor do we consider the interaction of the loop plasma with the background plasma and loop-loop interactions. We adopt a rather realistic field-line geometry to approximate the loop and do apply an enhanced plasma density along the loop, given the fact that in our approach, all conditions are applied and solutions are obtained along an individual field line. Unlike the kink mode in the standard theory as reviewed by Ruderman \& Erdélyi (2009); Nakariakov \& Verwichte (2005) for a cylindrical loop model of a finite radius and enhanced density, we are investigating the Alfvénic oscillations along an individual field line without such a columnated density structure.

\section{The Klein-Gordon equation}

Following McKenzie \& Hu (2010), we consider spatial variations only along the prescribed background field lines. We found an error in their original derivation and have presented the set of corrected formulas in a separate report (Webb et al. 2012). We repeat some of the relevant results below. We obtain the following wave equations for the perturbations $b_{\phi}$ and $u_{\phi}$ from the set of coupled Eqs. (2) and (3) (Webb et al. 2012), in which $V=B_{\theta} / \sqrt{\rho}$ is the poloidal Alfvén wave speed and $\partial / \partial \theta:=\mathrm{d} / \mathrm{d} \theta$ denotes the total derivative along an individual field line at a fixed time,

$$
\begin{aligned}
& \frac{\partial^{2} b_{\phi}}{\partial t^{2}}=\frac{V^{2}}{r^{2}}\left\{\frac{\partial^{2} b_{\phi}}{\partial \theta^{2}}-\frac{1}{L_{b}} \frac{\partial b_{\phi}}{\partial \theta}+\frac{b_{\phi}}{M_{b}}\right\} \\
& \frac{\partial^{2} u_{\phi}}{\partial t^{2}}=\frac{V^{2}}{r^{2}}\left\{\frac{\partial^{2} u_{\phi}}{\partial \theta^{2}}-\frac{1}{L_{u}} \frac{\partial u_{\phi}}{\partial \theta}+\frac{u_{\phi}}{M_{u}}\right\} .
\end{aligned}
$$

These fundamental equations are for transverse hydromagnetic waves (i.e., Alfvén waves) in an inhomogeneous media with a background axi-symmetric magnetic field. Here all the coefficients within the brackets are given by the following (all evaluated along $\boldsymbol{B}_{0}$ as functions of the co-latitude $\theta$ only), and are explicitly known for a given background magnetic field model and a density profile along the field line:

$$
\begin{aligned}
-\frac{1}{L_{b}} & =\frac{\mathrm{d}}{\mathrm{d} \theta} \ln \left(\frac{B_{\theta}}{\rho r}\right) \\
\frac{1}{M_{b}} & =\frac{r \rho}{B_{\theta}} \frac{\mathrm{d}}{\mathrm{d} \theta}\left(\frac{B_{\theta}}{\rho r} \frac{1}{l_{u}}\right)-\frac{1}{l_{b}^{2}} \\
-\frac{1}{L_{u}} & =\frac{\mathrm{d}}{\mathrm{d} \theta} \ln \left(\frac{B_{\theta}}{r}\right) \\
\frac{1}{M_{u}} & =\frac{r}{B_{\theta}} \frac{\mathrm{d}}{\mathrm{d} \theta}\left(\frac{B_{\theta}}{r} \frac{1}{l_{b}}\right)-\frac{1}{l_{u}^{2}},
\end{aligned}
$$

and

$\frac{1}{l_{b}}=-\frac{\mathrm{d}}{\mathrm{d} \theta} \ln (r \sin \theta)=-\frac{1}{l_{u}}$.

Equations (4) and (5) are of the form

$\frac{\partial^{2} \Phi}{\partial t^{2}}=\frac{V^{2}}{r^{2}}\left[\frac{\partial^{2} \Phi}{\partial \theta^{2}}-\frac{1}{\mathcal{L}} \frac{\partial \Phi}{\partial \theta}+\frac{1}{\mathcal{M}} \Phi\right]$

where

$\frac{1}{\mathcal{L}}=-\frac{\mathrm{d}}{\mathrm{d} \theta} \ln (F(\theta))$. 

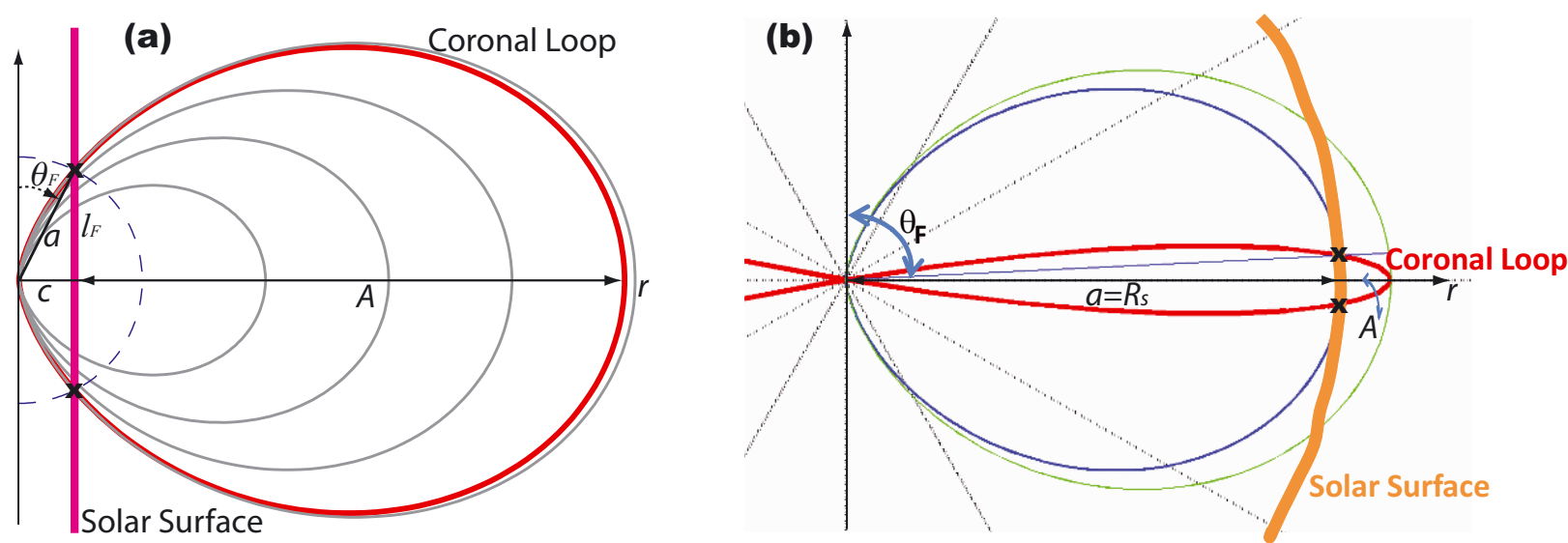

Fig. 1. a) The local-dipole loop geometry for a specific case (red line; radius $a$ is chosen as the distance from the center to one footpoint of colatitude $\theta_{\mathrm{F}}$ ): $\left.L a=A+c=4.91 a ; \mathbf{b}\right)$ an example of a stretched global dipole field line (red): the loop apex height $A=0.1 R_{\mathrm{S}}$ (solar radius), and $\theta_{\mathrm{F}}=\pi / 2-\pi / 64$. The blue line represents a standard dipole field line passing through the same footpoints of the red loop on solar surface, while the green one represents a dipole field line of the same apex height of the red loop. See Sect. 4 for details.

They can be transformed into the Klein-Gordon (KG) form through the substitution

$\Phi=\psi \exp \int \frac{\mathrm{d} \theta}{2 \mathcal{L}}=\psi f(\theta)$

to yield

$\frac{\partial^{2} \psi}{\partial t^{2}}+\omega_{\mathrm{c}}^{2} \psi=\frac{V^{2}}{r^{2}} \frac{\partial^{2} \psi}{\partial \theta^{2}}$

in which

$\omega_{\mathrm{c}}^{2}=\frac{V^{2}}{r^{2}}\left[\frac{1}{2 \mathcal{L}^{2}}\left(\frac{1}{2}+\frac{\mathrm{d} \mathcal{L}}{\mathrm{d} \theta}\right)-\frac{1}{\mathcal{M}}\right]$.

The so-called adiabatic-geometric growth/decay amplitude factor (McKenzie \& Hu 2010) in Eq. (13) is written

$f(\theta)=\exp \int \frac{\mathrm{d} \theta}{2 \mathcal{L}}=\frac{1}{F^{1 / 2}(\theta)}$.

It is clear that they possess the form

$f_{u}(\theta)=\left(\frac{B_{\theta}}{r}\right)^{-\frac{1}{2}}, \quad f_{b}(\theta)=\left(\frac{B_{\theta}}{\rho r}\right)^{-\frac{1}{2}}$,

for the velocity and magnetic field oscillation, respectively.

The quantity $\omega_{c}$, given by Eq. (15), in Eq. (14) is a cut-off frequency as is readily seen by taking a harmonic time variation $\propto \exp (i \omega t)$ for then Eq. (14) becomes

$\frac{\mathrm{d}^{2} \psi}{r^{2} \mathrm{~d} \theta^{2}}=-\frac{\left(\omega^{2}-\omega_{\mathrm{c}}^{2}\right)}{V^{2}} \psi \equiv-\kappa^{2} \psi$

Alternatively, by denoting $r \mathrm{~d} \theta=\frac{B_{\theta}}{B} \mathrm{~d} s$ (Webb et al. 2012), where the field-line segment is denoted by $\mathrm{d} s$, one may rewrite the above equation in the form $\left(V_{\mathrm{A}}=B / \sqrt{\rho}\right.$, the Alfvén speed $)$

$\frac{\mathrm{d}^{2} \psi}{\mathrm{d} s^{2}}=-\frac{\left(\omega^{2}-\omega_{\mathrm{c}}^{2}\right)}{V_{\mathrm{A}}^{2}} \psi$

A normal mode analysis (plane wave approximation) yields a dispersion relation $\omega^{2}=\omega_{\mathrm{c}}^{2}+k^{2} V_{\mathrm{A}}^{2}$. So the phase speed would generally take on values great than $V_{\mathrm{A}}$, and in the limit of $\omega \gg$ $\omega_{\mathrm{c}}$, a value equal to $V_{\mathrm{A}}$, the Alfvén speed.
As pointed out in McKenzie \& $\mathrm{Hu}$ (2010), an equation of this form possesses propagating-type solutions for $\omega>\omega_{\mathrm{c}}$ and evanescent solutions for $\omega<\omega_{\mathrm{c}}$. Since the cut-off frequency $\omega_{\mathrm{c}}$ varies with $\theta$ along the individual field line, both types of solutions may co-exist and be coupled. The solutions are also subject to boundary conditions at the footpoints of the field line. The final wave forms of the perturbations are obtained by multiplying the amplitude factor $f(\theta)$ of Eq. (16) with the corresponding KG equation solution.

In the cases to be examined below, the boundary conditions are chosen as $u_{\phi}=0$ at both footpoints of the loop. An additional condition $\mathrm{d} \psi / \mathrm{d} \theta=s$, an arbitrary constant, is chosen to enable the solutions of $\omega$ and $\psi$ simultaneously (an eigenvalue problem for $\omega$ ) via an ODE solver implemented in Matlab software package. The procedure is to solve the KG equation of $u_{\phi}$ with the complete set of boundary conditions to obtain an eigenfrequency $\omega$ and the solution of $u_{\phi}$. Then the magnetic field perturbation $b_{\phi}$ is obtained from Eq. (2) directly utilizing the known solution of $u_{\phi}$.

\section{Transverse oscillations in coronal loops}

We now consider the transverse oscillations in coronal loops where the KG equations can be applied. We examine two possible geometries of a coronal loop, all based on a standard axisymmetric dipole field model. The $\mathrm{KG}$ equations for such a dipole field have been worked out in Webb et al. (2012). Here we repeat some relevant results for completeness and extend the applications to solar coronal conditions.

A background dipole magnetic field is given by

$\boldsymbol{B}_{0} / B_{0}=2\left(\frac{a}{r}\right)^{3} \cos \theta \hat{r}+\left(\frac{a}{r}\right)^{3} \sin \theta \hat{\theta}$

where $a$ is the radius at which $B=B_{0}$ at the equator $\theta=\pi / 2$. The equation of a field line becomes

$\frac{\mathrm{d} r}{\mathrm{~d} \theta}=\frac{r B_{r}}{B_{\theta}}=2 r \cot \theta$

which yields

$r=L a \sin ^{2} \theta$ 
where $L a$ is the radius at which a given field line cuts the equator (so-called $L$ shell in Earth's dipole field). Then for this geometry, we have (Webb et al. 2012)

$\frac{1}{l_{b}}=-\frac{1}{l_{u}}=-3 \cot (\theta)$

and subsequently,

$$
\begin{aligned}
\frac{1}{L_{b}} & =7 \cot \theta+\frac{1}{\rho} \frac{\mathrm{d} \rho}{\mathrm{d} \theta} \\
\frac{1}{L_{u}} & =7 \cot \theta \\
\frac{1}{M_{u}} & =15 \cot ^{2} \theta+3 \\
\frac{1}{M_{b}} & =-3\left(11 \cot ^{2} \theta+1+\cot \theta \frac{\mathrm{d} \ln \rho}{\mathrm{d} \theta}\right) .
\end{aligned}
$$

It is clear that the KG equation and its solution depend on the density profile along the field line. For example, as in McKenzie $\& \mathrm{Hu}$ (2010), we assume $\rho \propto r^{-p}$ along the field line, i.e.,

$\rho=\rho_{0}(a / r)^{p}=\rho_{0} L^{-p} \sin ^{-2 p} \theta, \quad \rho_{0} \equiv$ const.

Subsequently, we obtain the cut-off frequency for the magnetic field perturbation in the form

$\omega_{\mathrm{c}}^{2}=\frac{\omega_{\mathrm{c} 0}^{2}}{L^{8-p} \sin ^{(16-2 p)} \theta}\left(\frac{13}{2}-p\right)\left[\left(\frac{15}{2}-p\right) \cos ^{2} \theta+\sin ^{2} \theta\right]$,

with $\omega_{\mathrm{c} 0}^{2}=\left(B_{0}^{2} / \rho_{0}\right) / a^{2}$. The corresponding cut-off frequency for the velocity perturbation is

$\omega_{\mathrm{c}}^{2}=\frac{\omega_{\mathrm{c} 0}^{2}}{4 L^{8-p} \sin ^{(16-2 p)} \theta}\left(\cos ^{2} \theta+2\right)$.

For wave frequencies $\omega$ in excess of (less than) the cut-off frequency the time harmonic KG Eq. (18) yields propagating (evanescent) modes. Often the cut-off frequency $\omega_{\mathrm{c}}$ exhibits a profile symmetric about the equator, with a minumum value at the equator and monotonically increasing values towards the footpoints (Webb et al. 2012). Thus for a determined eigenfrequency $\omega$ within the range of minimum and maximum cutoff frequency, we expect propagating modes equator-ward of where $\omega=\omega_{\mathrm{c}}$, and evanescent modes for $\omega<\omega_{\mathrm{c}}$ towards the footpoints.

The corresponding growth-decay factors are also obtained for both the magnetic field and velocity perturbations, respectively (Webb et al. 2012). For example, the amplification factor for the velocity perturbation becomes

$f_{u}=\frac{L^{2} a^{1 / 2}}{B_{0}^{1 / 2}} \sin ^{7 / 2} \theta$

Note that for a constant density along the field line ( $\rho \equiv$ const., i.e., $p=0$ in the above equations), the function $1 / \mathcal{L}$ becomes identical, and the factor $f=1 / \sqrt{F}$ differs by only a constant factor, in the equations of $b_{\phi}$ and $u_{\phi}$, as seen from Eqs. (6), (8), and (17).

\subsection{A local dipole-field loop}

First we consider a loop geometry of a local dipole field line near the solar surface. As illustrated in Fig. 1a, such a coronal loop may be represented by one field line of a standard dipole that has an arbitrary radius, $a$, and a symmetry axis not necessarily crossing the center of the Sun. In principle, certain parameters characterizing the loop geometry may be determined from remotesensing observations, such as the loop apex height, $A$, and the half-length between loop footpoints, $l_{\mathrm{F}}$ (see, e.g., Aschwanden et al. 2002). From Fig. 1a, the following is satisfied approximately (with the implication, $l_{\mathrm{F}} \ll R_{\mathrm{s}}$, the solar radius),

$a \approx \sqrt{c^{2}+l_{\mathrm{F}}^{2}}=\operatorname{Lasin}^{2} \theta_{\mathrm{F}} \approx(A+c) \frac{c^{2}}{a^{2}}$.

Therefore, it follows $a^{3}=(A+c) c^{2}$, i.e., $(A+c) c^{2}=\left(c^{2}+l_{\mathrm{F}}^{2}\right)^{\frac{3}{2}}$. Then the unknown parameter, $c$, the depth of the dipole center can be first determined by solving $(c>0)$

$2 A c^{5}+\left(A^{2}-3 l_{\mathrm{F}}^{2}\right) c^{4}-3 l_{\mathrm{F}}^{4} c^{2}-l_{\mathrm{F}}^{6}=0$,

with known parameters $A$ and $l_{\mathrm{F}}$ from observations. Then the other parameters can be determined subsequently.

Figure 1a shows one example for $A=0.05$ and $l_{\mathrm{F}}=0.01$ (all normalized by $R_{\mathrm{S}}$ ). The resulting parameters are $c=0.0051$, $L a=0.0551, a=0.011$, and $L=4.91$. The full loop length is about $88 \mathrm{Mm}$. The angle $\theta_{\mathrm{F}}=\tan ^{-1} c / l_{\mathrm{F}}=27^{\circ}$, and it is possible to fully determine the dipole magnetic field $\left(B_{0}\right)$ from the observation of $B$ at one loop footpoint $\left(\theta=\theta_{\mathrm{F}}\right)$ by

$B_{0}=B / \sqrt{4 \cos ^{2} \theta_{\mathrm{F}}+\sin ^{2} \theta_{\mathrm{F}}}$.

Of course the observation of $B$ is often only available on the photosphere where the loop footpoint is not always rooted. Therefore magnetic field extrapolation would be needed, in practice, to obtain the suitable measurement of $B$ at the loop footpoint. Under this geometry, all the formulations derived in Webb et al. (2012) and given above by Eqs. (20)-(31) remain unchanged. We choose a constant (corresponding to $p=0$ ) but relatively enhanced plasma number density along the loop, $n=10^{9} \mathrm{~cm}^{-3}$ (Aschwanden 2004; Aschwanden et al. 2002), and $B_{0}=100 \mathrm{G}$. In reality, these parameters may take a variable range of values and can be determined from observations. For example, the choice of $B_{0}$ is related to the magnetograph measurement $(B)$ at the loop footpoints by Eq. (34) with $\theta_{\mathrm{F}}$ derived from measurements by the procedure described above if the local dipole approximation is satisfactory. Therefore a value of 100 Gauss is reasonable, which corresponds to $B \approx 184 \mathrm{G}$, especially for a loop rooted in active regions of strong magnetic field.

Figure 2 shows the cut-off frequency and the factor $f(\theta)$ of $u_{\phi}$ for various $L$ values corresponding to the loop geometries shown in Fig. 1a. They show significant variations along the loop: the cut-off frequency changes more than one order of magnitude, while the amplitude factor changes up to tens of times, especially for larger $L$ values. The wave forms of different modes are similar to those in Webb et al. (2012), but are of different eigenfrequencies, $\omega$. The fundamental mode for this particular case is shown in Fig. 3. The spatial profiles of higher-order harmonics are given in Fig. 4 for both $b_{\phi}$ and $u_{\phi}$. They all exhibit the feature of a propagating-type solution near the center and an evanescent type towards the footpoints. The oscillation frequency increases with the increasing order of the harmonics while the amplitude decreases. The corresponding time periods $(P=2 \pi / \omega)$ of the 

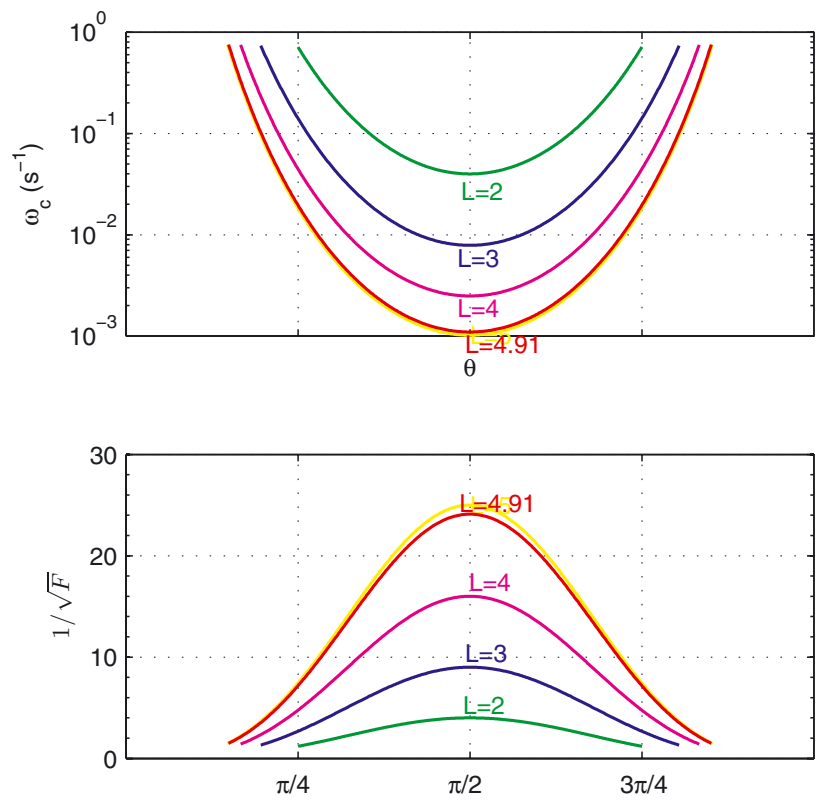

Fig. 2. The cut-off frequency $\omega_{\mathrm{c}}$ (with $B_{0}=100$ Gauss, $a=0.011 R_{\mathrm{s}}$, and $n=10^{9} \mathrm{~cm}^{-3}$ ), and the adiabatic growth/decay factor as a function of $\theta$ for various $L$ values ( $\rho \equiv$ const. along the loop) for the transverse velocity oscillation.
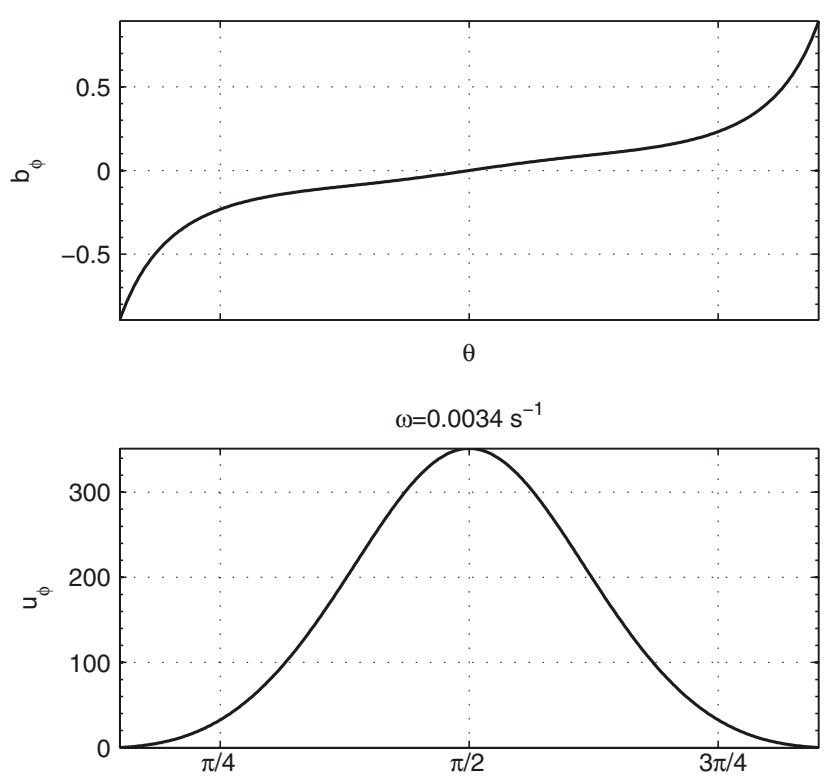

Fig. 3. Wave form from the solution of the KG equation for the local dipole field line $(L=4.91)$ : the fundamental mode. Both $u_{\phi}$ and $b_{\phi}$ are of arbitrary units.

oscillation range from the longest $31 \mathrm{~min}$ of the fundamental mode to about 1.3 min of the multiple-order mode in Fig. 4d. The time period of the fundamental mode is on the same order of magnitude as the high-end of the range of the values reported from observations (Aschwanden et al. 2002).

Figure 5 shows the variation of the fundamental mode frequency with respect to the set of $L$ values for different density profiles. The corresponding loop lengths are also indicated by the right axis. It is seen that the frequency decreases with increasing $L$ value, but increases with exponent $p$. The results seem to be consistent with Oliver et al. (1993), who examined the MHD waves in a potential magnetic arcade structure of translation symmetry in a Cartesian coordinate system. To further visualize our result and to make connection with actual observations, we illustrate, in Fig. 6, the spatial profiles of oscillating magnetic field lines at the moment of maximum amplitudes, corresponding to the fundamental, 1st-order, and 2nd-order harmonics, respectively. They show certain resemblance to actual observations (e.g., Aschwanden et al. 2002). In reality, one may foresee the difficulty in mode identification in terms of the number of nodes, especially that associated with higher-order modes due to projection effect etc.

\subsection{A stretched global dipole-field loop}

Now we consider an alternative model of the coronal loop which may better mimic the observed geometry under certain circumstances. Gibson \& Low (1998), in an attempt to develop a theoretical model for Coronal Mass Ejections (CMEs) based on MHD and a self-similar solution, utilized a radialstretching transformation to better simulate the observed rather complex geometry and morphology of CME cavities with embedded magnetic field. The resulting configuration maintains the solenoidal condition of the magnetic field and a magnetohydrostatic equilibrium. The approach was able to transform a simpler and regular magnetic field configuration to a more complex one, for example, from a circle to a water-drop shape that better mimics coronagraph image of a CME. This approach suits our purpose well. Following Gibson \& Low (1998), we apply their stretching transformation $r \rightarrow \Lambda$ to a global dipole field line (symmetry axis always crossing the center of the Sun)

$\Lambda=k r+r_{\mathrm{d}}$,

where parameters $k$ and $r_{\mathrm{d}}$ are constant. Then the resulting field line equation for such a stretched dipole field becomes

$r=L a \sin ^{2} \theta-\frac{r_{\mathrm{d}}}{k} \cos ^{2} \theta$

And the magnetic field components are (Gibson \& Low 1998, where lower-case $b$ denotes the original dipole field, and $\left.r_{\mathrm{c}} \equiv r_{\mathrm{d}} / k\right)$ :

$$
\begin{aligned}
B_{r} & =\left(\frac{\Lambda}{r}\right)^{2} b_{r}(\Lambda, \theta, \phi) \\
& =\frac{2 B_{0} a^{3}}{k} \frac{\cos \theta}{r^{2}\left(r+r_{\mathrm{c}}\right)} \\
B_{\theta} & =\frac{\Lambda}{r} \frac{\mathrm{d} \Lambda}{\mathrm{d} r} b_{\theta}(\Lambda, \theta, \phi) \\
& =\frac{B_{0} a^{3}}{k} \frac{\sin \theta}{r\left(r+r_{\mathrm{c}}\right)^{2}} .
\end{aligned}
$$

It is clear that for the parameter $r_{\mathrm{d}} \equiv 0$ and $k=1$, the magnetic field geometry returns to a standard dipole, and here $a \equiv R_{\mathrm{s}}$.

Figure $1 \mathrm{~b}$ shows one example of such a coronal loop (red line) for the chosen parameters: $L a=1.1 a, A=0.1 a$, and $l_{\mathrm{F}} / a=\pi / 64=\pi / 2-\theta_{\mathrm{F}}$ (keeping $k=1$ hereafter). It deviates from a standard dipole field line and is non-potential. The full loop length is about $164 \mathrm{Mm}$. All the formulas related to the $\mathrm{KG}$ equations of the perturbations can be derived for this particular field-line geometry. They are listed below $\left(r_{\mathrm{c}} \equiv r_{\mathrm{d}} / k\right)$ :

$$
\begin{aligned}
& \frac{1}{l_{u}}=\cot \theta\left(3+\frac{2 r_{\mathrm{c}}}{r}\right) \\
& \frac{1}{l_{b}}=-\frac{1}{l_{u}},
\end{aligned}
$$


(a)
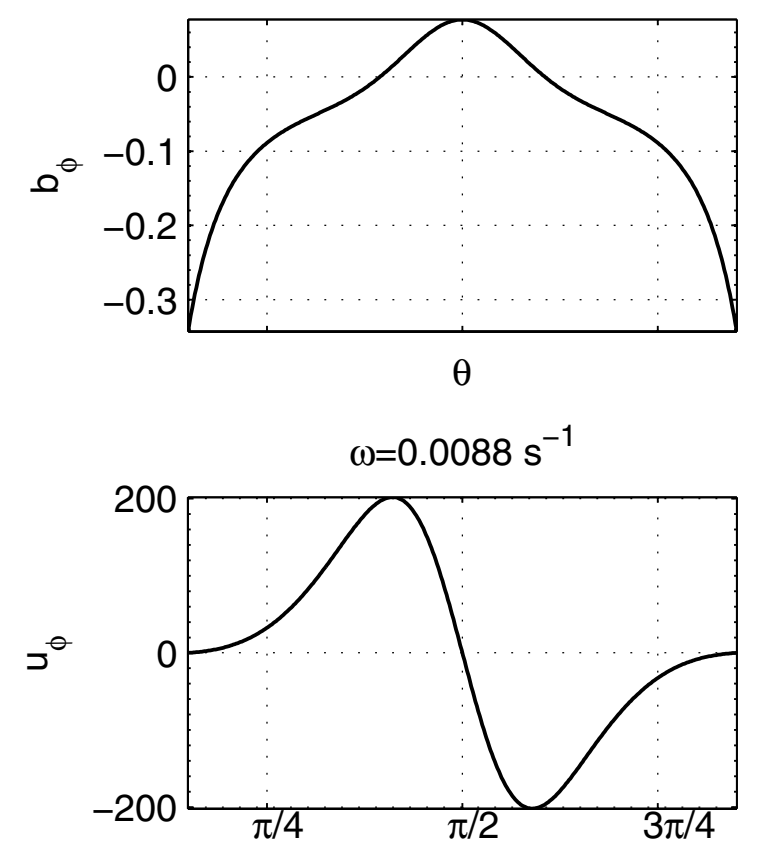

(c)
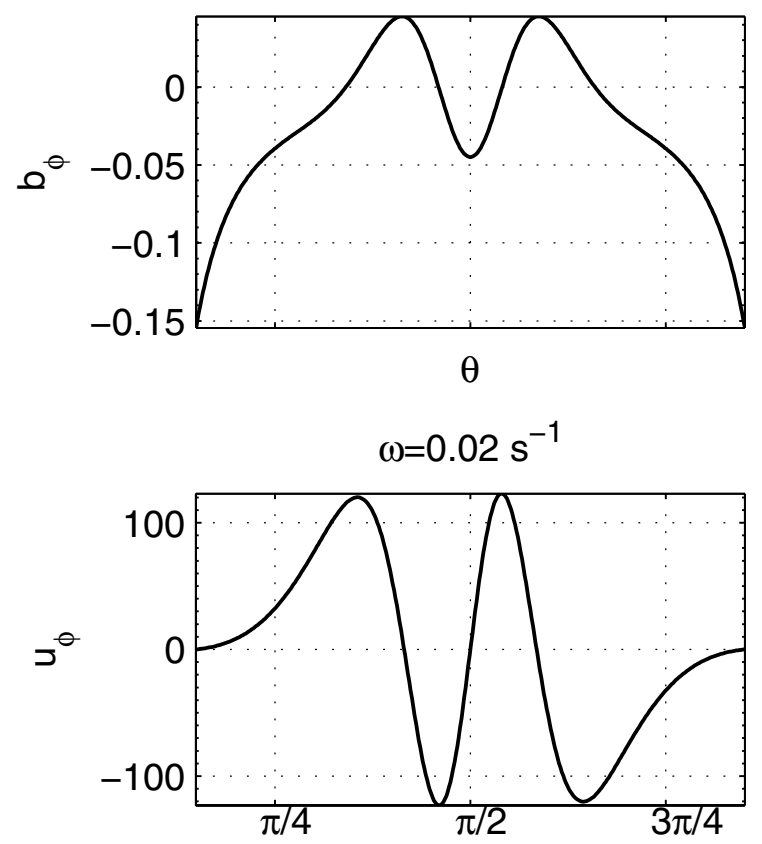

(b)
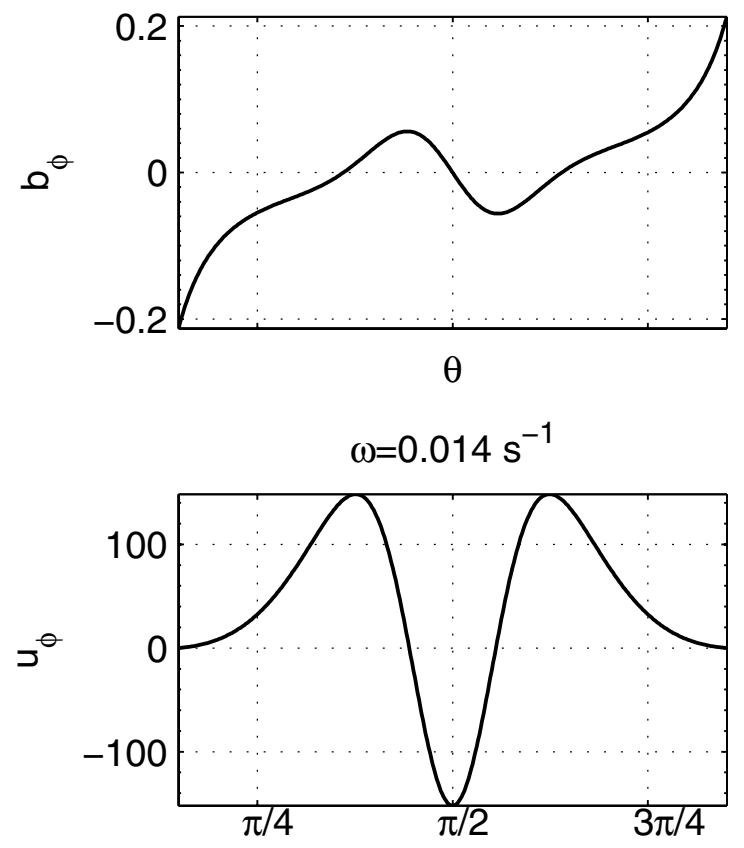

(d)

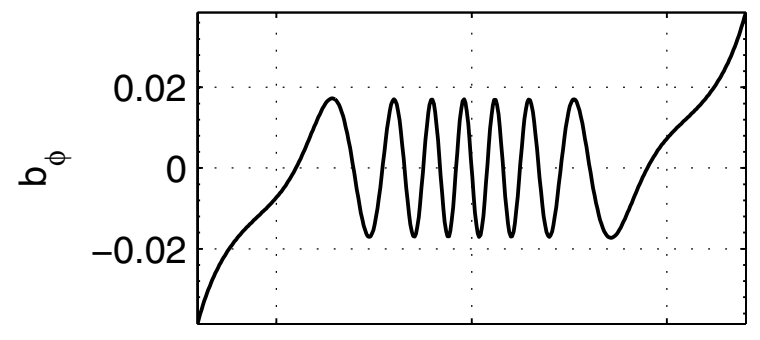

$\theta$

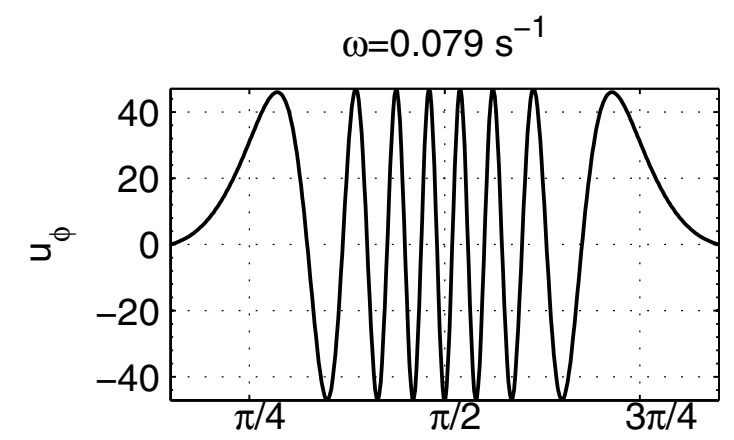

Fig. 4. Wave forms from the harmonic solutions of the KG equation of multiple orders: a) 1st, b) 2nd, c) 3rd, and d) multiple orders. Both $u_{\phi}$ and $b_{\phi}$ are of arbitrary units. The eigen-frequency $\omega$ is denoted in each panel.

and

$\frac{1}{L_{u}}=\cot \theta\left(7+\frac{4 r_{\mathrm{c}}}{r}\right)$

$\frac{1}{L_{b}}=\frac{1}{L_{u}}+\frac{\mathrm{d} \ln \rho}{\mathrm{d} \theta}$

$\frac{1}{M_{u}}=\frac{1}{L_{u}} \frac{1}{l_{u}}-\frac{\mathrm{d}}{\mathrm{d} \theta}\left(\frac{1}{l_{u}}\right)-\frac{1}{l_{u}^{2}}$

$\frac{1}{M_{b}}=-\frac{1}{M_{u}}-\frac{2}{l_{b}^{2}}+\frac{1}{l_{b}} \frac{\mathrm{d} \ln \rho}{\mathrm{d} \theta}$.
It can be checked that all the above equations return to the forms corresponding to a standard dipole as reported in Webb et al. (2012) and Eqs. (23)-(27) for $r_{\mathrm{c}} \equiv 0$ (without stretching).

Figure 7 shows the variations of both $\omega_{\mathrm{c}}$ and $f(\theta)$ for this particular case of a stretched dipole field loop (with $B_{0}=100 \mathrm{G}, a \equiv$ $R_{\mathrm{S}}$, and constant number density along the loop, $n=10^{9} \mathrm{~cm}^{-3}$ ). Note that the angular extent of the loop becomes very limited on a global scale in this case. But the overall behavior remains similar with extreme values still at the equator. However the range of variations (the ratio of maximum over minimum values) is 


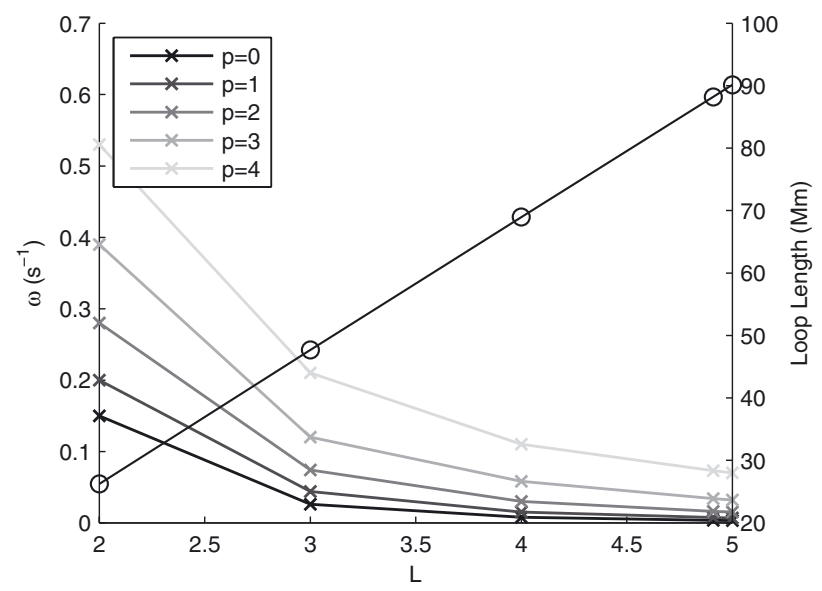

Fig. 5. The variation of the fundamental mode frequencies as a function of $L$ for different density profiles (exponent $p$ ). The loop length for each $L$ value (denoted by a circle) is given by the right axis.
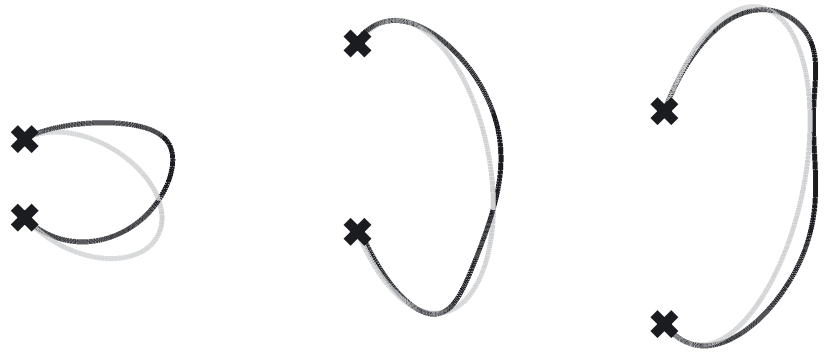

Fig. 6. Spatial profiles of the oscillating magnetic field lines (of maximum amplitude; black curves) for the fundamental, 1st-order, and 2ndorder harmonics (from left to right), corresponding to Fig. 3, Fig. 4a and $b$, respectively. The gray curve is the same background field line at rest and the cross symbol denotes the footpoint. The view angle of each panel is altered separately in order to optimize the visualization of each oscillation pattern and amplitude.

significantly less than the previous case in Sect. 4.1. Figures 8 and 9 show the wave forms and the corresponding oscillation frequencies for the fundamental, and higher-order mode harmonics. Again the frequencies increase with higher orders. But the wave forms contain the propagating-type solutions only since $\omega>\omega_{\mathrm{c}}$ is satisfied throughout the latitudinal interval of the loop in this particular case. Therefore their wave forms are closer to a sinusoidal form than the previous case without pronounced decaying behavior towards the footpoints. The oscillation periods range from $17 \mathrm{~min}$ for the fundamental mode to about $45 \mathrm{~s}$ for the multiple-order mode in Fig. 9d. The fundamental mode period falls within the observed range of periods of transverse oscillations in coronal loops (e.g., Aschwanden et al. 2002). Figure 10 again shows the oscillating magnetic field lines of different modes for this particular case. They are Alfvénic $\left(\omega \gg \omega_{\mathrm{c}}\right)$ standing waves between the footpoints with increasing number of nodes $(0,1$, and 2 , respectively), increasing oscillation frequency, and decreasing oscillation amplitude.

\section{Summary and discussion}

In summary, we present a hydromagnetic theory of the transverse magnetic field and velocity perturbations in an axisymmetric background magnetic field geometry which applies to one particular ("horizontal") transverse oscillation of solar
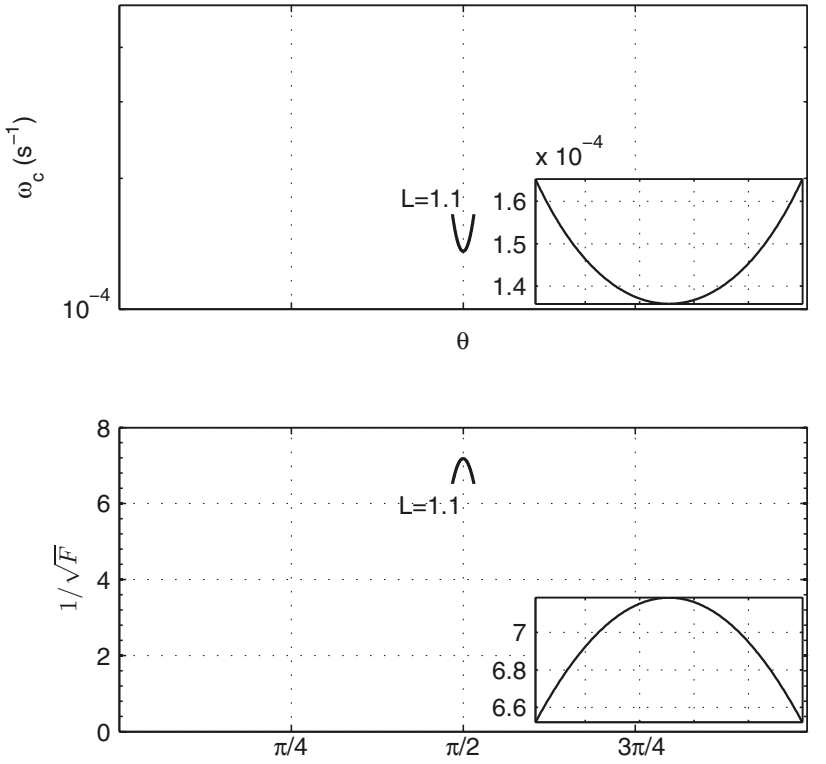

Fig. 7. Same format as Fig. 2 for the stretched dipole field loop $(L=$ 1.1). The insets show the same curves over the loop interval $\theta \in\left[\frac{\pi}{2}-\right.$ $\left.\frac{\pi}{64}, \frac{\pi}{2}+\frac{\pi}{64}\right]$ only.
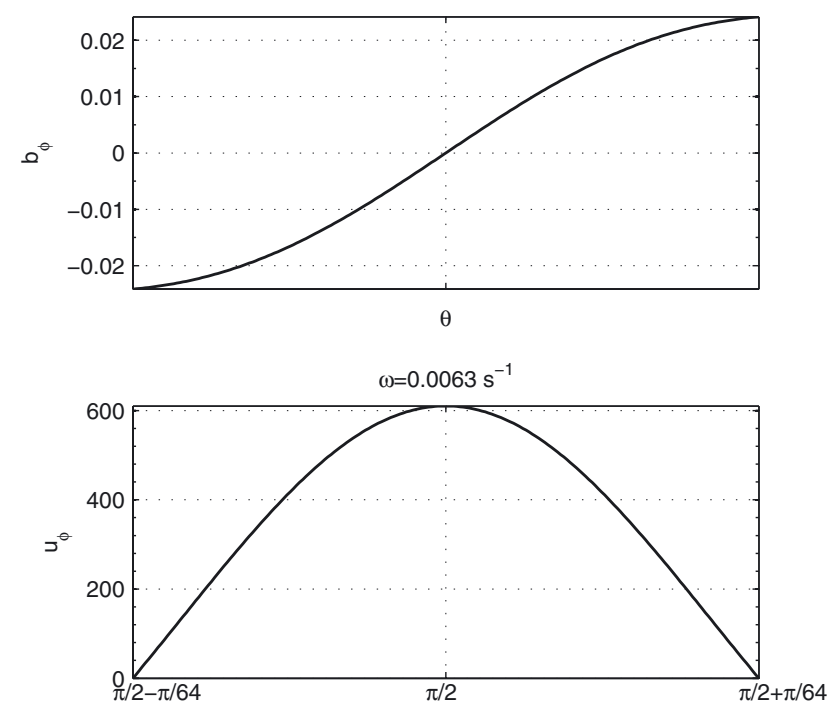

Fig. 8. Wave form from the solution of the KG equation for the stretched dipole: the fundamental mode. Both $u_{\phi}$ and $b_{\phi}$ are of arbitrary units.

coronal loops. The corresponding wave equations are cast into a special $\mathrm{KG}$ form that is represented by an ordinary differential equation after a wave amplitude transformation with harmonic time variations $\left(\propto \mathrm{e}^{\mathrm{i} \omega t}\right)$. We illustrate its applications to coronal loop oscillations by adopting two background field-line models that may mimic real coronal loop geometries. We obtain the oscillation periods $(P=2 \pi / \omega)$ of the fundamental and higher-order harmonics for the two models. They range from tens of minutes for the fundamental mode to $<1 \mathrm{~min}$ for multiple-order mode, with the chosen set of magnetic field and plasma parameters, for the chosen loops. The fundamental mode periods are generally on the same order of magnitude as the observed periods of transverse coronal loop oscillations (e.g., Aschwanden et al. 2002; Goossens et al. 2002), especially considering the variability in the parameter values of our model (e.g., $\omega \propto \omega_{\mathrm{c} 0}=B_{0} /\left(a \sqrt{\rho}_{0}\right)$ ), if our approximation warrants 
(a)

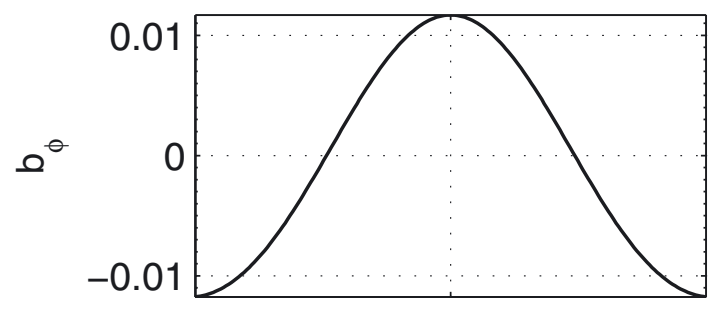

$\theta$

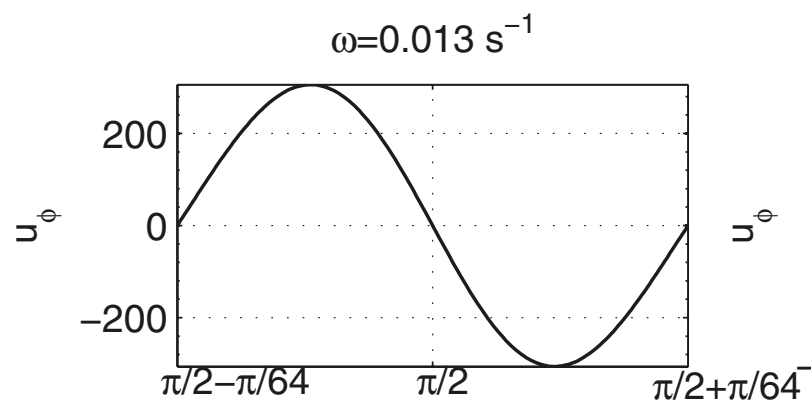

(c)

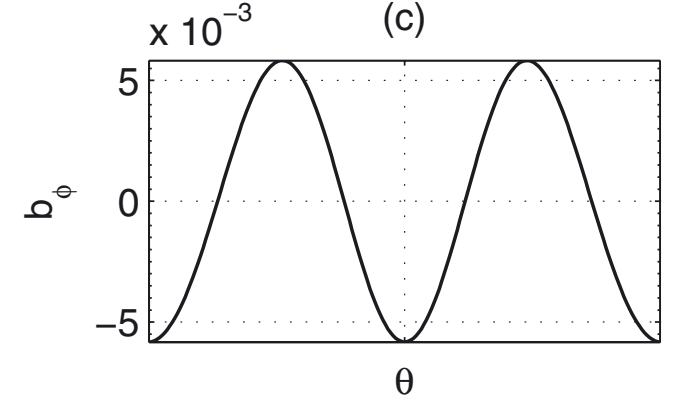

$\omega=0.026 \mathrm{~s}^{-1}$

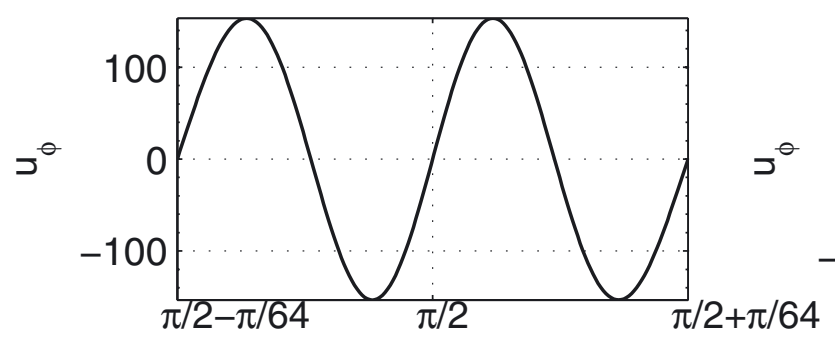

$\times 10^{-3}$

(b)

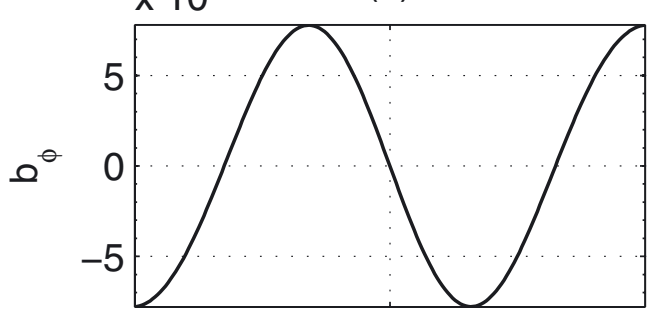

$\theta$

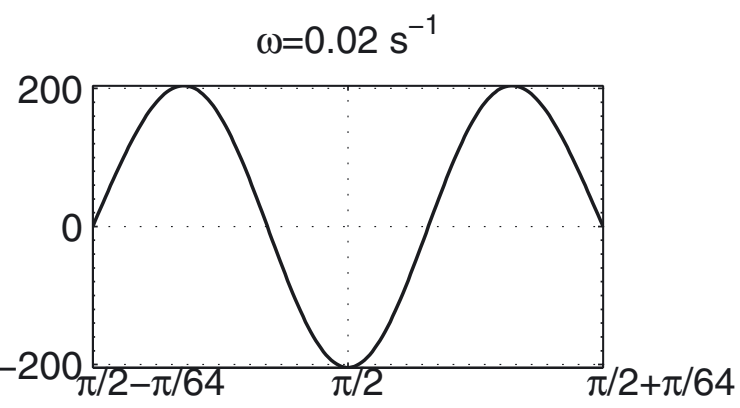

(d)

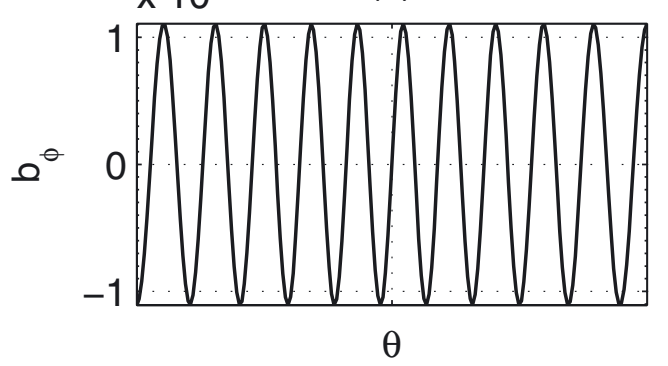

$\omega=0.14 \mathrm{~s}^{-1}$

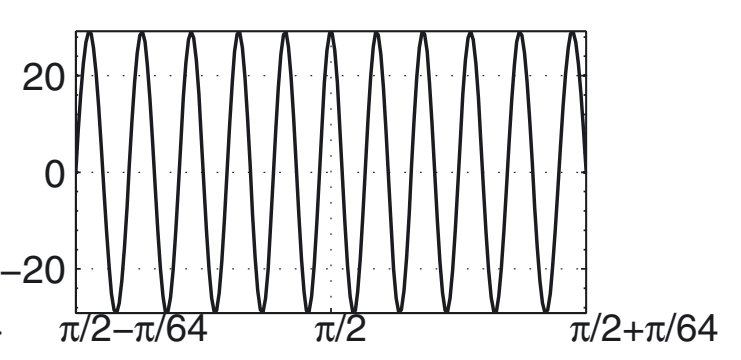

Fig. 9. Wave forms from the harmonic solutions of the KG equation of multiple orders: a) 1 st, b) 2 nd, c) 3rd, and d) multiple orders. Both $u_{\phi}$ and $b_{\phi}$ are of arbitrary units.

such a comparison with some emission-line observations. The observations of higher-order harmonic oscillations (overtones) were reported before (e.g., Andries et al. 2009). Direct comparison with theory is desirable, but would still be challenging, given the ambiguity in distinguishing different spatial profiles. Some wave forms show spatially coupled solutions of a propagating type near the equator $\left(\omega>\omega_{\mathrm{c}}\right)$ and an evanescent type towards the ends $\left(\omega<\omega_{\mathrm{c}}\right)$, while others exhibit purely propagating type throughout the loop interval, owing to the behavior of the cutoff frequency, $\omega_{\mathrm{c}}$, of different field-line geometries. These wave forms, characteristic of the KG equations, yet need to be further studied and compared with observations. We will pursue these detailed studies in future work. For example, different density profiles seem to lead to different eigen-frequencies that fit different observations. One caveat, as we discussed in Sect. 2, is that the distinction between the magnetic field line and the loop itself as manifested from emission-line observations is not addressed in our model.

In practice, the choice of boundary conditions has to be guided by observations. As we pointed out earlier (McKenzie $\& \mathrm{Hu} 2010$ ), the observation-based boundary conditions will assign proper physical units to $u_{\phi}$ and $b_{\phi}$, which will enable further comparison of our results with observations in terms of oscillation amplitude and exact spatial wave forms. For example, the asymmetric spatial profile of loop oscillations may be simply modeled by choosing asymmetric loop footpoints, i.e., asymmetric interval of loop co-latitude $\theta$, and/or different boundary conditions at different footpoints. For real event, a better model 

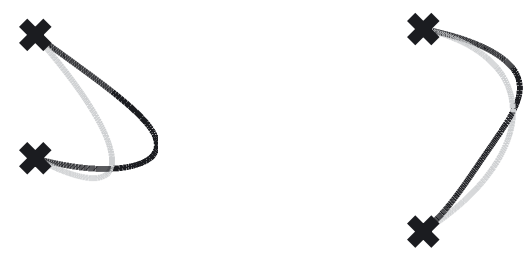

Fig. 10. Spatial profiles of the oscillating magnetic field lines (of maximum amplitude) for the fundamental, 1st-order, and 2nd-order harmonics (from left to right), corresponding to Figs. 8, 9a and b, respectively. Format is the same as Fig. 6.

for coronal loop (magnetic field line) is probably the combination of the two cases presented in Sect. 4, i.e., a stretched local dipole-field line, which also allows a non-potential field topology (Gibson \& Low 1998). The simplest case would be shifting the center of the global dipole (with fixed $a=R_{\mathrm{S}}$ ) away from the solar center to account for a loop plane not crossing the center of the Sun. Then the analysis in Sect. 4.2 readily applies, and observations would facilitate the determination of various parameters associated with the analysis outlined in previous sections. In the present study, we limited our investigations to one family of theoretical solutions subject to one particular set of boundary conditions that yield oscillation periods consistent with observations, but of small and arbitrary wave amplitudes. One limitation of our theory is that we do not address the damping of the oscillations in time that is commonly observed (e.g., Aschwanden et al. 2002; Wang et al. 2002), partially owing to the fact that we do not incorporate finite loop thickness and various other effects in our model (Ruderman \& Erdélyi 2009; Goossens et al. 2002). However it might be possible for us to simulate such behavior by employing time-varying boundary conditions. Of course the underlying mechanisms for wave excitation and dissipation would remain separate subjects that need to be addressed.

One interesting feature of our solutions is the determination of the magnetic field along the loop once the constant parameter $B_{0}$ is determined from, e.g., the magnetograph measurement, given the underlying magnetic field model assumed. Alternatively, if the oscillation frequency $\omega$ is well determined from observations, a similar eigen-value problem for the unknown parameter $B_{0}\left(\kappa^{2}\right.$ in Eq. (18)) can be constructed for a specific field-line model. Thus the magnetic field along an individual loop can be determined by examining the corresponding loop oscillation characteristics, provided that the observed loop geometry fits the magnetic field model satisfactorily. This would provide an additional tool for "coronal seismology".

Acknowledgements. We thank the anonymous referee for constructive comments and insight which have significantly improved our understanding and presentation of this work. H.Q. and G.M.W. acknowledge NASA grant NNX10AG03G and NSF grant AGS-1062050 for partial support. One of the authors (JFMcK) acknowledges support from the Pei-Ling Chan Chair of Physics in the University of Alabama in Huntsville. J.F.McK. also acknowledges support from the NRF of South Africa.

\section{References}

Andries, J., Van Doorsselaere, T., Roberts, B., et al. 2009, Space Sci. Rev., 149, 3

Aschwanden, M. 2004, Physics of the solar corona: An Introduction, Ch. 7 (Chichester UK: Praxis Publishing Ltd, and Berlin: Springer), 283

Aschwanden, M., De Pontieu, B., Schrijver, C. J., \& Title, A. 2002, Sol. Phys., 206, 99

Gibson, S. E., \& Low, B. C. 1998, ApJ, 493, 460

Goossens, M., Andries, J., \& Aschwanden, M. J. 2002, A\&A, 394, L39

McKenzie, J. F., \& Hu, Q. 2010, Ann. Geophys., 28, 1

Miyagoshi, T., Yokoyama, T., \& Shimojo, M. 2004, PASJ, 56, 207

Nakariakov, V. M., \& Verwichte, E. 2005, Liv. Rev. Sol. Phys., 2, 3 , http : //www . livingreviews . org/lrsp-2005-3

Ofman, L. 2009, Space Sci. Rev., 149, 153

Oliver, R., Ballester, J. L., Hood, A. W., \& Priest, E. R. 1993, A\&A, 273, 647

Ruderman, M. S., \& Erdélyi, R. 2009, Space Sci. Rev., 149, 199

Ruderman, M. S., \& Scott, A. 2011, A\&A, 529, A33

Schrijver, C. J., Aschwanden, M. J., \& Title, A. 2002, Sol. Phys., 206, 69

Selwa, M., \& Ofman, L. 2010, ApJ, 714, 170

Terradas, J., Oliver, R., \& Ballester, J. L. 2006, ApJ, 650, 91

Van Doorsselaere, T., Debosscher, A., Andries, J., \& Poedts, S. 2004, A\&A, 424, 1065

Van Doorsselaere, T., Verwichte, E., \& Terradas, J. 2009, Space Sci. Rev., 149, 299

Wang, T., Solanki, S. K., Curdt, W., Innes, D. E., \& Dammasch, I. E. 2002, ApJ, 574, L101

Wang, T., Solanki, S. K., Curdt, W., et al. 2003, A\&A, 406, 1105

Wang, T., Solanki, S. K., \& Selwa, M. 2008, A\&A, 489, 1307

Webb, G. M., McKenzie, J. F., Hu, Q., \& Zank, G. P. 2012, JGR, submitted 